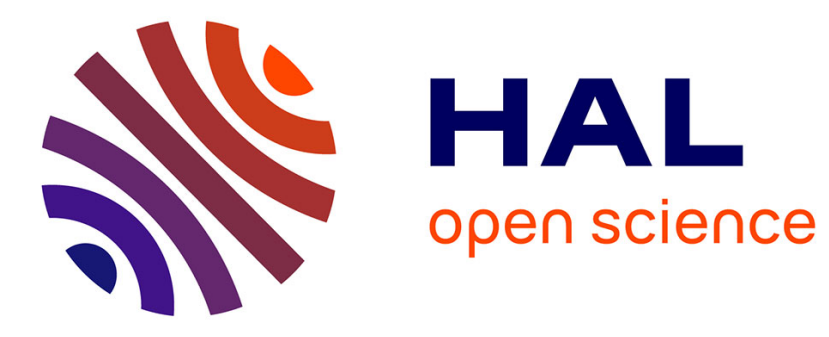

\title{
Generalization of GLRT-Based Magnetic Anomaly Detection
}

Pascal Pepe, Steeve Zozor, Laure-Line Rouve, Jean-Louis Coulomb, Christine Serviere, Jean Muley

\section{- To cite this version:}

Pascal Pepe, Steeve Zozor, Laure-Line Rouve, Jean-Louis Coulomb, Christine Serviere, et al.. Generalization of GLRT-Based Magnetic Anomaly Detection. EUSIPCO 2015 - 23th European Signal Processing Conference, Aug 2015, Nice, France. hal-01200898

\section{HAL Id: hal-01200898 \\ https://hal.science/hal-01200898}

Submitted on 17 Sep 2015

HAL is a multi-disciplinary open access archive for the deposit and dissemination of scientific research documents, whether they are published or not. The documents may come from teaching and research institutions in France or abroad, or from public or private research centers.
L'archive ouverte pluridisciplinaire HAL, est destinée au dépôt et à la diffusion de documents scientifiques de niveau recherche, publiés ou non, émanant des établissements d'enseignement et de recherche français ou étrangers, des laboratoires publics ou privés. 


\title{
GENERALIZATION OF GLRT-BASED MAGNETIC ANOMALY DETECTION
}

\author{
P. Pepe $e^{1,2,3}$, S. Zozor ${ }^{1}$, L.-L. Rouve ${ }^{2}$, J.-L. Coulomb ${ }^{2}$, C. Servière ${ }^{1}$, J. Muley ${ }^{3}$ \\ ${ }^{1}$ GIPSA-lab \\ ${ }^{2}$ G2Elab \\ Université Grenoble Alpes \\ Grenoble, France \\ Université Grenoble Alpes \\ Grenoble, France \\ ${ }^{3}$ DCNS \\ Lorient, France
}

\begin{abstract}
Magnetic anomaly detection (MAD) refers to a passive method used to reveal hidden magnetic masses and is most commonly based on a dipolar target model. This paper proposes a generalization of the MAD through a multipolar model that provides a more precise description of the anomaly and serves a twofold objective: to improve the detection performance, and to widen the variety of detectable targets. The dipole detection strategy - namely an orthonormal decomposition of the anomaly followed by a generalized likelihood ratio test - is hence revisited in the multipolar case. The performance are assessed analytically and the relevance of this generalization is demonstrated on multipolar scenarios.
\end{abstract}

Index Terms - Magnetic anomaly detection, spherical harmonic expansion, orthonormal basis functions

\section{INTRODUCTION}

Magnetic anomaly detection (MAD) refers to a passive method used for anti-submarine warfare, and is typically fulfilled by an aircraft carrying a magnetometer and flying over a maritime area, searching for hidden ferromagnetic targets. This approach is based on the well known fact that the presence of a ferromagnetic mass in the Earth's magnetic field causes a modification of the field in its surrounding area, termed magnetic anomaly. This spatial deformation of the field, if significant enough to be detected from an airborne system, will most likely be due to a submarine.

The studies on MAD found in the literature can be split into two categories. The first one finds its roots in the seventies with noticeable contributions established by regarding the target as a magnetic dipole, thus providing a mathematical modeling of the anomaly that paved the way for a statistical detection strategy $[1,2]$. This approach is still drawing significant interest today [3-6]. The second category appeared more recently and consists in methods focused on the magnetic background nature instead of target-based models $[7,8]$.

The study presented in this paper focuses on contributing to the first category. The aim is to propose a generalization of the target-based approach that overcomes the limits imposed by the convenient, but nonetheless restrictive dipolar approximation. For this purpose, we present a multipolar target model in section 2 in comparison with the dipolar model. The detection strategy is revisited in section 3 and the performance are analytically assessed in section 4 . Finally, the impact of our generalization and the gain of performance are illustrated through multipolar scenarios in section 5 .

\section{EXPRESSION OF THE MAGNETIC ANOMALY}

\subsection{Definition of a multipolar target model}

In a typical scenario, the sensor is assumed to be moving along a straight line at a constant speed $V$ and at a constant altitude while the target is assumed stationary (the opposite configuration is however completely analogous). The classical approach consists in considering the target as a magnetic dipole defined by its static moment $\mathbf{m}$ (represented as a $3 \times 1$ column vector). Its presence in the Earth's magnetic field induces an anomaly $\mathbf{H}$ that can be expressed at any point by [9]:

$$
\mathbf{H}(\mathbf{r})=\frac{3\left(\mathbf{m}^{t} \mathbf{r}\right) \mathbf{r}-r^{2} \mathbf{m}}{4 \pi r^{5}},
$$

where $\mathbf{r}$ is the Cartesian coordinate vector of the point considered (the origin of the system is at the center of the target), $r=\|\mathbf{r}\|$, and ${ }^{t}$ denotes the transpose of vectors and matrices. Equation (1) is only valid at a long distance between the source and the sensor and is therefore limited to specific contexts. Instead, we suggest a representation of the target based on a spherical harmonic expansion of the magnetic field, as commonly used in electromagnetic problems that require to model an unknown source [10-13] and also similarly in many other research fields $[14,15]$. This expansion is obtained by solving the Laplace equation in spherical coordinates, which gives the following expression of the anomaly $[9,10]$ :

$$
\begin{aligned}
\mathbf{H}(\mathbf{r})=-\operatorname{grad}\left(\sum_{n=1}^{\infty}\right. & \frac{1}{r^{n+1}} \sum_{m=0}^{n}\left(a_{n, m} \cos m \phi\right. \\
& \left.\left.\quad+b_{n, m} \sin m \phi\right) \mathcal{P}_{n}^{m}(\cos \theta)\right),
\end{aligned}
$$

where $\mathbf{r}$ is here alternatively represented by its spherical coordinates $(r, \theta, \phi)$ defined so that $r$ is the radial distance (as 
previously), $\theta \in[0, \pi]$ is the polar angle, and $\phi \in]-\pi, \pi]$ is the azimuthal angle. The integers $n$ and $m$ respectively denote a decomposition order and its associated degree. The scalars $a_{n, m}$ and $b_{n, m}$ are called the spherical harmonic coefficients, and are characteristic of the magnetic properties of the target. The terms $\mathcal{P}_{n}^{m}$ are the associated Legendre polynomials. Equation (2) gives a more precise description of the target anomaly than (1) by taking into account the dipole moment (first-order term) as well as the higher-order moments (quadrupole, octupole, etc.). As implied within (2), the influence of each decomposition order $n$ decreases as $1 / r^{n+2}$, which means that far from the sources the higher orders become negligible and eventually non-measurable. In practice, the infinite sum is therefore truncated to an arbitrary decomposition order $L$ that should match the target at best.

A particular position, called the closest point of approach (CPA), is reached by the sensor at the instant $t_{0}$ when it is at the closest to the target, with $D$ being the corresponding distance. At any instant $t$, the position of the sensor can be specified by the dimensionless variable $u=\frac{\left(t-t_{0}\right) V}{D}$. Through several mathematical considerations involving the gradient operation and the associated Legendre polynomials (see [16, 17]), we have rewritten (2) for any point along the sensor track as:

$$
\mathbf{H}(u)=\sum_{n=1}^{L} \sum_{i=0}^{n+1} \mathbf{c}_{n, i} \frac{u^{i}}{\left(1+u^{2}\right)^{n+\frac{3}{2}}},
$$

where the functions $f_{n, i}(u)=u^{i} /\left(1+u^{2}\right)^{n+\frac{3}{2}}$ are only parametrized by the parameters of the CPA (namely $D$ and $t_{0}$ ), and the $3 \times 1$ vector coefficients $\mathbf{c}_{n, i}$ depend on the magnetic parameters of the target (given by $a_{n, m}$ and $b_{n, m}$ ) and do not depend on time. The dipolar assumption is a first-order approximation that consists in choosing $L=1$, which leads to the expression established in [1,2] and widely discussed since then [3-6]. From this perspective, we can see that our result (3) encompasses and generalizes the dipolar approach.

\subsection{Orthonormal basis function decomposition}

In (3), the $L(L+5) / 2$ functions $f_{n, i}$ are linearly dependent if $L>1$. Indeed, for any $i \in \mathbb{N}$ and any $n \in \mathbb{N}^{*}$, we have $f_{n, i}+f_{n, i+2}=f_{n-1, i}$. However, by recursively using this relation we have reduced the set of $L(L+5) / 2$ functions to a linearly independent set of only $2 L+1$ functions, which consists of $f_{0,0}$, to which two more functions $f_{n, 0}$ and $f_{n, 1}$ are added for each successive decomposition order considered $n=(1,2, \ldots, L)$. From there, we can perform an orthonormalization in order to build a set of orthonormal basis functions (OBF) denoted $\left(e_{i}\right)_{i \in \llbracket 0,2 L \rrbracket}$. In this way, (3) can be rewritten as:

$$
\mathbf{H}(u)=\sum_{i=0}^{2 L} \mathbf{a}_{i} e_{i}(u),
$$

where the vector coefficients $\mathbf{a}_{i}$ are characteristic of the multipole in this basis.

\section{GLRT-BASED DETECTION STRATEGY}

\subsection{Detection problem}

We consider the acquisition of $N$ samples of the anomaly (4), which are concatenated in order to build the $3 \times N$ matrix $\mathbf{S}$ defined by:

$$
\mathbf{S}=\mathbf{A}_{L} \mathbf{E}_{L},
$$

where $\mathbf{A}_{L}$ is the $3 \times(2 L+1)$ matrix of coefficients so that $\mathbf{A}_{L}=\left[\mathbf{a}_{0}, \ldots, \mathbf{a}_{2 L}\right]$, and $\mathbf{E}_{L}$ is the $(2 L+1) \times N$ matrix consisting of $N$ samples from every OBF. Depending on its technology, the sensor used may provide a $3 \mathrm{D}$ or a $1 \mathrm{D}$ information. In order to describe both contexts within one single formalism, as done in [5], the target signal is thereafter represented by the $d \times N$ matrix $\mathbf{s}$, where $d=3$ or 1 , either defined directly from (5) or by $\mathbf{H}_{0}^{t} \mathbf{S} /\left\|\mathbf{H}_{0}\right\|$, which is the projection of the anomaly on the Earth's magnetic field $\mathbf{H}_{0}$, as approximately obtained with a scalar (1D) magnetometer.

The MAD problem consists in deciding from $N$ measurements whether a signal is present or if only noise is observed. Assuming that the Earth's magnetic field has been previously removed from the measurements, MAD leads to a classical binary hypothesis testing problem [18]:

$$
\left\{\begin{array}{c}
\mathcal{H}_{0} \text { (absence of target) }: \mathbf{x}=\mathbf{w} \\
\mathcal{H}_{1} \text { (presence of target) }: \mathbf{x}=\mathbf{s}+\mathbf{w}
\end{array}\right.
$$

where $\mathbf{x}$ is the observation and $\mathbf{w}$ is noise. From there, we define $P_{D}=\operatorname{Pr}\left(\mathcal{H}_{1} \mid \mathcal{H}_{1}\right)$ the probability of detection and $P_{F A}=\operatorname{Pr}\left(\mathcal{H}_{1} \mid \mathcal{H}_{0}\right)$ the probability of false alarm. In the sequel, the target signal is always assumed deterministic, and the noise is assumed Gaussian, isotropic and white. The random matrix $\mathbf{w}$ has therefore a matrix variate normal distribution with zero mean and covariance matrix $\sigma^{2} \mathbf{I}_{d} \otimes \mathbf{I}_{N}$, where $\sigma^{2}$ denotes the spatio-temporal variance of each noise sample, and $\otimes$ is the Kronecker product. This is alternatively denoted $\mathbf{w} \sim \mathcal{N}_{d, N}\left(\mathbf{0}, \sigma^{2} \mathbf{I}_{d} \otimes \mathbf{I}_{N}\right)$. The probability density function (PDF) of $\mathbf{w}$ is then given by [19]:

$$
p(\mathbf{w})=\left(2 \pi \sigma^{2}\right)^{-\frac{d N}{2}} \exp \left(-\frac{\operatorname{tr}\left(\mathbf{w}^{t} \mathbf{w}\right)}{2 \sigma^{2}}\right) .
$$

Following the Neyman-Pearson approach, we aim at maximizing $P_{D}$ under a $P_{F A}$ constraint.

\subsection{Known or assumed known signal basis}

In practice, even in a cooperative context, the signal of the target can scarcely ever be known because the inherent magnetic parameters of the target are completely unknown. In this subsection, we will assume that the parameters of the signal basis ( $D$ and $t_{0}$ ) are known, as well as $L$, the order of the multipole. This means that $\mathbf{E}_{L}$ is known while $\mathbf{A}_{L}$ is unknown. 
The approach consists here in a generalized likelihood ratio test (GLRT) [18]:

$$
\frac{p\left(\mathbf{x} ; \widehat{\mathbf{A}}_{L}, \mathcal{H}_{1}\right)}{p\left(\mathbf{x} ; \mathcal{H}_{0}\right)} \underset{\mathcal{H}_{0}}{\stackrel{\mathcal{H}_{1}}{\gtrless}} \gamma\left(P_{F A}\right),
$$

where $\widehat{\mathbf{A}}_{L}$ is the maximum likelihood estimator (MLE) of $\mathbf{A}_{L}$, and $p\left(\mathbf{x} ; \widehat{\mathbf{A}}_{L}, \mathcal{H}_{1}\right)$ and $p\left(\mathbf{x} ; \mathcal{H}_{0}\right)$ denote the PDF of $\mathbf{x}$ under each hypothesis. The problem of estimating $\mathbf{A}_{L}$ from (5) in the particular case of white Gaussian noise is well known and, similarily to $[2,3]$ we have shown that the coefficient matrix that maximizes the likelihood $p\left(\mathbf{x} ; \widehat{\mathbf{A}}_{L}, \mathcal{H}_{1}\right)$ is the projection of $\mathbf{x}$ onto the basis $\mathbf{E}_{L}$ [20]:

$$
\widehat{\mathbf{A}}_{L}=\mathbf{x} \mathbf{E}_{L}^{t} .
$$

By using (9) and (7) in (8) and taking the logarithm of both sides, the receiver writes:

$$
\Lambda_{\mathrm{OBF}-L}(\mathbf{x})=\operatorname{tr}\left(\widehat{\mathbf{A}}_{L}^{t} \widehat{\mathbf{A}}_{L}\right)=\left\|\widehat{\mathbf{A}}_{L}\right\|^{2},
$$

which will thereafter be referred to as the OBF-based receiver of order $L$, or in a more compact way as the OBF- $L$ receiver. It is worth mentionning that (10) is also the energy of the projection. While the dipolar approach already led to OBF-1, we have now access to a wider range of receivers that can be applied to targets more complex than a dipole.

\subsection{Unknown signal basis}

We can go a step further by considering that even the signal basis is unknown. The knowledge of the signal basis is related to the three following parameters: $D, t_{0}$ and $L$.

The case of $D$ was addressed in [2] by considering a bank of receivers built for different possible values of the parameter, and later in [5] by using a genetic algorithm instead.

The parameter $t_{0}$ is not a concern in a real-time scenario, because in practice windows of the observation are projected on centered OBF with a fixed length (see section 5).

Estimating $L$ can be seen as a model selection problem. Among the available methods, the Akaike information criterion (AIC) [21] is quite convenient in our approach. Indeed, AIC depends on the number of parameters to adjust (the elements of $\mathbf{A}_{L}$ ) but also on the maximized value of the likelihood of the model, and $\widehat{\mathbf{A}}_{L}$ has been specifically chosen as the MLE. AIC is therefore straightforwardly expressed as:

$$
\begin{gathered}
A I C_{L}=2 d(2 L+1)+2 d N \log \left(2 \pi \sigma^{2}\right) \\
+\frac{1}{\sigma^{2}}\left(\|\mathbf{x}\|^{2}-\left\|\widehat{\mathbf{A}}_{L}\right\|^{2}\right) .
\end{gathered}
$$

The value of $L$ that minimizes $A I C_{L}$ also corresponds to the model that minimizes the information loss, and thus in the same way to the maximal order of the multipole that should match the target at best. The application of this criterion is illustrated in section 5 .

\section{PERFORMANCE OF THE DETECTORS}

This section aims at analytically assessing the theoretical performance of the OBF- $L$ detectors. In the context of this paper, we will assume that the parameter of the signal basis $D$ is known and rather focus on the influence of $L$, as it is the parameter of main interest in this multipolar approach.

\subsection{OBF- $L$ detector when $L$ is known}

First of all, we consider the case of the OBF- $L$ detector where $L$ is the correct multipolar order. In other words, we pick the OBF- $L$ detector for a target that is indeed best described by a multipole of order $L$. The coefficient matrix $\mathbf{A}_{L}$ is estimated by projection of the observation onto the appropriate basis $\mathbf{E}_{L}$ according to (9). Then, under $\mathcal{H}_{k}(k=0$ or 1$)$ :

$$
\widehat{\mathbf{A}}_{L}=k \mathbf{s} \mathbf{E}_{L}^{t}+\mathbf{w} \mathbf{E}_{L}^{t},
$$

which means that $\widehat{\mathbf{A}}_{L} \sim \mathcal{N}_{d, 2 L+1}\left(k \mathbf{s} \mathbf{E}_{L}^{t}, \sigma^{2} \mathbf{I}_{d} \otimes \mathbf{I}_{2 L+1}\right)$ (see [19]). The OBF- $L$ receiver (10) over $\sigma^{2}$ is thus basically the sum of the squares of $(2 L+1) d$ independent standard normal random variables. Therefore,

$$
\frac{\operatorname{tr}\left(\widehat{\mathbf{A}}_{L}^{t} \widehat{\mathbf{A}}_{L}\right)}{\sigma^{2}} \sim \begin{cases}\chi_{(2 L+1) d}^{2} & \text { under } \mathcal{H}_{0} \\ \chi_{(2 L+1) d}^{2}\left(\frac{\left\|\mathbf{A}_{L}\right\|^{2}}{\sigma^{2}}\right) & \text { under } \mathcal{H}_{1}\end{cases}
$$

where $\chi_{\nu}^{2}$ and $\chi_{\nu}^{2}(\lambda)$ denote the chi-squared distribution with $\nu$ degrees of freedom and, for the latter, a noncentrality parameter $\lambda$. The term $E N R=\left\|\mathbf{A}_{L}\right\|^{2} / \sigma^{2}$ defines the energyto-noise ratio. By noting $F_{\chi_{\nu}^{2}}$ and $F_{\chi_{\nu}^{2}(\lambda)}$ respectively the cumulative distribution functions of $\chi_{\nu}^{2}$ and $\chi_{\nu}^{2}(\lambda)$, the theoretical performance of the OBF- $L$ detector are given by:

$$
P_{D}=1-F_{\chi_{(2 L+1) d}^{2}}(E N R)\left(F_{\chi_{(2 L+1) d}^{2}}^{-1}\left(1-P_{F A}\right)\right) .
$$

\subsection{Consequence of picking an erroneous order}

The target is still best defined by a multipole of order $L$, but now, instead of using the appropriate detector, we use the OBF- $L^{\prime}$ detector, where $L^{\prime} \neq L$. In this case, the coefficient matrix $\mathbf{A}_{L}$ is estimated by projection of the observation onto the erroneous basis $\mathbf{E}_{L^{\prime}}$, and thus, under $\mathcal{H}_{k}$, $\widehat{\mathbf{A}}_{L^{\prime}} \sim \mathcal{N}_{d, 2 L^{\prime}+1}\left(k \mathbf{s E}_{L^{\prime}}^{t}, \sigma^{2} \mathbf{I}_{d} \otimes \mathbf{I}_{2 L^{\prime}+1}\right)$. As a consequence, following the same reasoning as in the previous subsection, the performance of the detector become:

$$
P_{D}^{\prime}=1-F_{\chi_{\left(2 L^{\prime}+1\right) d}^{2}}\left(E N R^{\prime}\right)\left(F_{\chi_{\left(2 L^{\prime}+1\right) d}^{2}}^{-1}\left(1-P_{F A}\right)\right),
$$

where $E N R^{\prime}=\left\|\mathbf{s} \mathbf{E}_{L^{\prime}}^{t}\right\|^{2} / \sigma^{2}=\left\|\mathbf{A}_{L} \mathbf{E}_{L} \mathbf{E}_{L^{\prime}}^{t}\right\|^{2} / \sigma^{2}$, which can be seen as an "erroneous ENR". With $L^{\prime}<L$, we have $E N R^{\prime}<E N R$. On the other hand, if $L^{\prime}>L$, then $E N R^{\prime}=E N R$, but too many degrees of freedom are allowed. Both cases lead to a loss of performance, as illustrated in the next section. 


\section{SIMULATION RESULTS}

\subsection{Influence of the multipolar order}

We will successively consider different multipolar scenarios by adjusting the values of the coefficients in $\mathbf{A}_{L}$. A dipole is represented by the first three coefficients (see section 2), and can also be seen as a multipole of pure order 1. More realistically, several coefficients will be involved in the description of the target as we will most likely face multipoles of non-pure orders. This is summed up in figure 1.

Most of the signal energy is spread within a time span of $10 D / V$ around $t_{0}$. The signal-to-noise ratio (SNR) can be set by adjusting the noise level in this window. In each of the following scenarios, we generate an observation of 10000 samples in a time span of $50 \mathrm{D} / \mathrm{V}$ with $t_{0}$ at the center. Windows of the observation are then projected on centered OBF with a fixed length corresponding to a time span of $10 \mathrm{D} / \mathrm{V}$.

1. The first scenario is chosen as a "didactic case" consisting in a target represented by a multipole of pure order 2 .

2. The second one consists in a more realistic target represented by a multipole of non-pure order 3 . We adjust the coefficients in $\mathbf{A}_{L}$ so that $70 \%$ of the signal energy is contained in the first three coefficients, $20 \%$ in the next two, and $10 \%$ in the other two. The first component of the signal of this target and its noisy observation are shown in figure 2 in arbitrary units with a SNR of $-15 \mathrm{~dB}$, alongside the output of the OBF-3 receiver.

Figure 3 shows the performance of OBF- $L$ detectors when applied to those two targets. In both cases, $P_{F A}$ is constrained to $10^{-4}$ and the performance are assessed either from the theoretical results (13) and (14) or from Monte-Carlo (MC) simulations. First of all, the didactic scenario with a multipole of pure order 2 (figure 3.a) shows that the best performance are obtained with the OBF-2 detector, which matches the target at best. As expected, the target is non-detectable by the OBF-1 detector. The OBF-3 detector is also able to detect the target with however a loss of performance as compared to the OBF2 detector, allowing too many degrees of freedom. Then for the more realistic scenario with a multipole of non-pure order 3 , figure 3.b shows the performance of the OBF-1 and OBF3 detectors. In this case, the performance are also evaluated by Monte-Carlo simulations, which perfectly match the theoretical expectations. Even though most of the signal energy $(70 \%)$ is contained in the first-order coefficients, the OBF-3 detector is still the one that leads to the best performance.

Figure 4 shows the AIC values for different model orders in both scenarios, with a SNR of $-15 \mathrm{~dB}$. The minimal value of the AIC is reached for the order that matches the target at best, which is also the detector order that leads to the best performance.

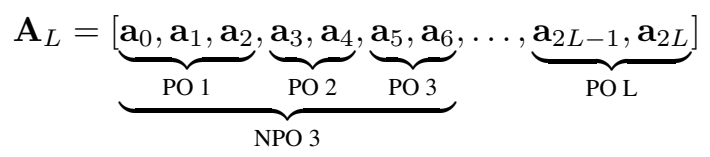

Fig. 1. Layout of the coefficient matrix $\mathbf{A}_{L}$. Illustration of the notions of pure order (PO) and non-pure order (NPO).

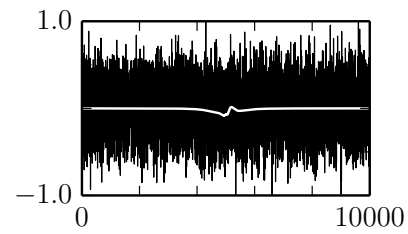

(a) Noisy observation

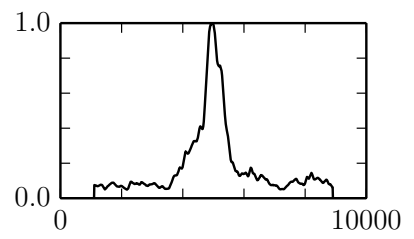

(b) OBF-3 receiver
Fig. 2. Representations of the first component of the signal of the second target (white) over its noisy observation for $S N R=-15 \mathrm{~dB}$ (black) (a) and of the output of the OBF-3 receiver calculated in a sliding window of 2000 samples (b).

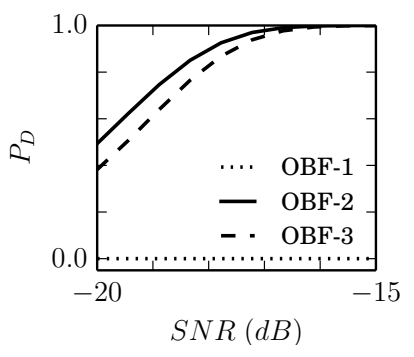

(a) Target 1

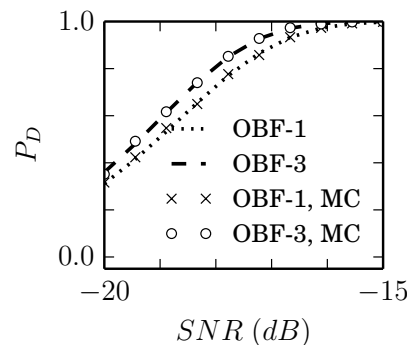

(b) Target 2
Fig. 3. Performance of the OBF- $L$ detectors when applied to the first (a) and to the second target (b).

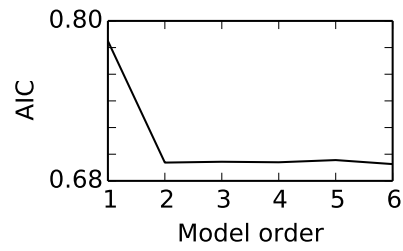

(a) Target 1

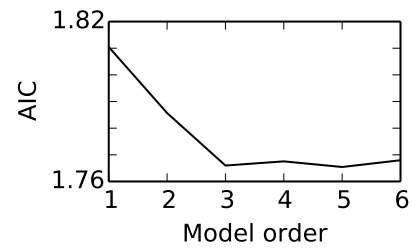

(b) Target 2
Fig. 4. AIC when applied to the first (a) and to the second target (b) for different model orders and $S N R=-15 \mathrm{~dB}$.

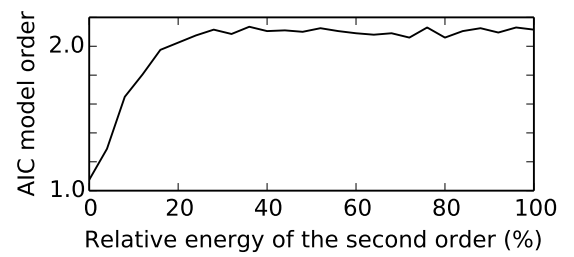

Fig. 5. Influence of the energy distribution on the AIC-based model selection for a target of order $2(S N R=-15 \mathrm{~dB})$. 


\subsection{Influence of the relative order magnitudes}

We consider now successive targets of order 2 , for which the coefficients in $\mathbf{A}_{L}$ are adjusted so that the relative energy of the second order varies from 0 to $100 \%$. Figure 5 shows the results of the AIC-based model selection (averaged on 100 simulations) for each of these targets (with $S N R=-15 \mathrm{~dB}$ ). It shows that very little energy is needed in the second order for OBF-2 to become more relevant than OBF-1.

\section{CONCLUSION}

In this paper, the MAD approach has been generalized to the detection of magnetic multipoles. While the "classical" MAD is restricted to the detection of dipoles, our multipolar approach is applicable to a wider variety of targets and, as such, could be used in more scenarios and applications than the typical context of the MAD. When the target is indeed more complex than a simple dipole, the multipolar approach provides a clear gain of performance, which illustrates the relevance of the method, especially in a detection context where even a slight gain could mean much.

\section{REFERENCES}

[1] E. P. Loane, "Speed and depth effects in magnetic anomaly detection," Memorandum report, ADA081329, unclassified document, Oct. 1976.

[2] R. Blanpain, Traitement en temps réel du signal issu d'une sonde magnétométrique pour la détection d'anomalies magnétiques, Ph.D. thesis, INP Grenoble, France, 1979.

[3] R. Otnes, "Static magnetic dipole detection using vector linear prediction, Anderson functions, and blockbased adaptive processing," in Proc. OCEANS 2007, Aberdeen, Scotland, 2007.

[4] A. Sheinker, L. Frumkis, B. Ginzburg, N. Salomonski, and B.-Z. Kaplan, "Magnetic anomaly detection using a three-axis magnetometer," IEEE Trans. Magn., vol. 45, no. 1, pp. 160-167, Jan. 2009.

[5] S. Zozor, L.-L. Rouve, G. Cauffet, J.-L. Coulomb, and H. Henocq, "Compared performances of MF-based and locally optimal-based magnetic anomaly detection," in Proc. EUSIPCO 2010, Aalborg, Denmark, 2010, pp. 149-153.

[6] H. Zhang and M.-Y. Xia, "Magnetic anomaly detection for simultaneous moving target and magnetometer," in Proc. APCAP 2014, Harbin, China, 2014, pp. 884-888.

[7] A. Sheinker, N. Salomonski, B. Ginzburg, L. Frumkis, and B.-Z. Kaplan, "Magnetic anomaly detection using entropy filter," Meas. Sci. Technol., vol. 19, no. 4, pp. 045205, Feb. 2008.
[8] A. Sheinker, B. Ginzburg, N. Salomonski, P. A. Dickstein, L. Frumkis, and B.-Z. Kaplan, "Magnetic anomaly detection using high-order crossing method," IEEE Trans. Geosci. Remote Sens., vol. 50, no. 4, pp. 1095-1103, Apr. 2012.

[9] J. D. Jackson, Classical Electrodynamics, John Wiley \& Sons, Inc., 3rd edition, 2007.

[10] L.-L. Rouve, L.-A. Schmerber, O. Chadebec, and A. Foggia, "Optimal magnetic sensor location for spherical harmonic identification applied to radiated electrical devices," IEEE Trans. Magn., vol. 42, no. 4, pp. 1167-1170, Apr. 2006.

[11] L.-A. Schmerber, Identification et caractérisation de sources électromagnétiques, Application à la discrétion des moteurs de propulsion navale, Ph.D. thesis, INP Grenoble, France, 2006.

[12] T. S. Nguyen, J.-M. Guichon, O. Chadebec, P. Labie, and J.-L. Coulomb, "Ships magnetic anomaly computation with integral equation and fast multipole method," IEEE Trans. Magn., vol. 47, no. 5, pp. 1414-1417, May 2011.

[13] K. Jerbi, J. C. Mosher, S. Baillet, and R. M. Leahy, "On MEG forward modelling using multipolar expansions," Phys. Med. Biol., vol. 47, no. 4, pp. 523-555, Feb. 2002.

[14] S. Parimal, S. M. Cramer, and S. Garde, "Application of a spherical harmonics expansion approach for calculating ligand density distributions around proteins," J. Phys. Chem. B, vol. 118, no. 46, pp. 13066-13076, Sept. 2014.

[15] A. C. Simmonett, F. C. Pickard, H. F. Schaefer, and B. R. Brooks, "An efficient algorithm for multipole energies and derivatives based on spherical harmonics and extensions to particle mesh Ewald," J. Chem. Phys., vol. 140, no. 18, pp. 184101, May 2014.

[16] I. S. Gradshteyn and I. M. Ryzhik, Table of Integrals, Series, and Products, Academic Press, 7th edition, 2007.

[17] F. W. J. Olver, D. W. Lozier, R. F. Boisvert, and C. W. Clark, NIST Handbook of Mathematical Functions, Cambridge University Press, New York, NY, 2010.

[18] S. M. Kay, Fundamentals of Statistical Signal Processing: Detection theory, Prentice-Hall PTR, 1998.

[19] A. K. Gupta and D. K. Nagar, Matrix Variate Distributions, CHAPMAN \& HALL/CRC, 2000.

[20] S. M. Kay, Fundamentals of Statistical Signal Processing: Estimation theory, Prentice-Hall PTR, 1993.

[21] H. Akaike, "A new look at the statistical model identification," IEEE Trans. Automat. Contr., vol. 19, no. 6, pp. 716-723, Dec. 1974. 